\begin{tabular}{|c|c|}
\hline Title & Design and characterization of nonlinear functions for the transmission of a small signal with non-Gaussian noise \\
\hline Author(s) & Kasai, Seiya; Tadokoro, Y ukihiro; Ichiki, A kihisa \\
\hline Citation & $\begin{array}{l}\text { Physical Review E, 88(6), } 062127 \\
\text { https://doi.org/10.1103PhysRevE.88.062127 }\end{array}$ \\
\hline Issue Date & 2013-12-16 \\
\hline Doc URL & http:/hdl.handle.net/2115/54592 \\
\hline Rights & @2013 A merican Physical Society \\
\hline Type & article \\
\hline File Information & PRE886_062127.pdf \\
\hline
\end{tabular}

Instructions for use 


\title{
Design and characterization of nonlinear functions for the transmission of a small signal with non-Gaussian noise
}

\author{
Seiya Kasai, ${ }^{1, *}$ Yukihiro Tadokoro, ${ }^{2}$ and Akihisa Ichiki ${ }^{3}$ \\ ${ }^{1}$ Graduate School of Information Science and Technology, and Research Center for Integrated Quantum Electronics, Hokkaido University, \\ Sapporo 060-0814, Japan \\ ${ }^{2}$ Toyota Central R\&D Labs., Inc., Nagakute, Aichi 480-1192, Japan \\ ${ }^{3}$ Green Mobility Collaborative Research Center, Nagoya University, Nagoya 464-8603, Japan
}

(Received 20 September 2013; published 16 December 2013)

\begin{abstract}
We design nonlinear functions for the transmission of a small signal with non-Gaussian noise and perform experiments to characterize their responses. Using statistical design theory [A. Ichiki and Y. Tadokoro, Phys. Rev. E 88, 012124 (2013)], a static nonlinear function is estimated from the probability density function of the given noise in order to maximize the signal-to-noise ratio of the output. Using an electronic system that implements the optimized nonlinear function, we confirm the recovery of a small signal from a signal with non-Gaussian noise. In our experiment, the non-Gaussian noise is a mixture of Gaussian noises. A similar technique is also applied to the optimization of the threshold value of the function. We find that, for non-Gaussian noise, the response of the optimized nonlinear systems is better than that of the linear system.
\end{abstract}

DOI: 10.1103/PhysRevE.88.062127 PACS number(s): 05.40.Ca, 05.45.-a, 89.70.-a, 84.30.-r

\section{INTRODUCTION}

Nonlinear systems often exhibit beneficial responses to a small, noisy signal. These include stochastic resonance (SR) [1-5], noise-induced linearization [6], noise-enhanced stability [7], and noise-assisted logic operations [8]. The response is obviously correlated with the nonlinearity and noise characteristics, and understanding this relationship has been a key issue in both science and engineering. There have been several studies on the noise-characteristic dependence of the response [9-12], but in most cases, the behavioral approach has been used for analysis due to the difficulties of a full analytical treatment of a nonlinear function and noise. Recently, Ichiki and Tadokoro reported an analytical approach to finding a static nonlinear function that is optimized to enhance a small signal buried in a non-Gaussian noise [13]. Their approach is based on Fisher's optimization, so we call it functional Fisher's optimization (FFO). It systematically deduces an optimal nonlinear function from the characteristics of the given noise. In this paper, we investigate the FFO-based design of a nonlinear function for the transmission of a small signal with non-Gaussian noise. We demonstrate it for two scenarios: the full design of a nonlinear function and the optimization of a parameter in a threshold function. We show that the design approach is feasible, not just for a full design, but also for optimizing the parameters in a given function. For the non-Gaussian noise, we used a noise mixture generated by a linear combination of a pseudorandom bit sequence (PRBS) and a Gaussian noise. This is a typical model for ambient noise that consists of a mixture of an artificial electromagnetic signal from wireless communications and natural noise [14-16]. It is also used in image restoration $[17,18]$. We used electronic devices to implement the nonlinear functions that we designed and then compared their responses to those of a conventional linear system.

\footnotetext{
*Corresponding author: kasai@ rciqe.hokudai.ac.jp
}

\section{DESIGN OF A NONLINEAR FUNCTION}

The FFO approach to the design of a static function $f(x)$ for the transfer of a small, noisy signal is to find the $f$ that maximizes the signal-to-noise ratio (SNR) of the output on the basis of Fisher's optimization [13]. We assume that $\xi(t)$ is white noise, the signal $S(t)$ is sufficiently small compared to the noise, and the noise component in the output is given by the variance of the output. Applying the linear response theory to the output $f(S+\xi)$, the output SNR is given by

$$
\mathrm{SNR}=\frac{\left\langle f^{\prime}(\xi)\right\rangle^{2} P_{S}}{\left\langle f^{2}(\xi)\right\rangle-\langle f(\xi)\rangle^{2}},
$$

where $\langle f\rangle$ denotes the expected value of $f$ and $P_{S}$ is the signal power of $S$. An optimum $f$ is estimated from $\delta \mathrm{SNR} / \delta f=0$. The optimum $f$ is then given by

$$
f=a-b \frac{\partial \ln \rho(x)}{\partial x},
$$

where $\rho$ is a probability density function (PDF) of the noise and $a$ and $b$ are constants $(b>0)$. The optimum function depends only on the noise, not on the signal, because the input signal intensity is assumed to be sufficiently small compared to the noise intensity. This assumption is valid when the contribution of the input signal to the denominator of Eq. (1) is ignorable, namely, $\left\langle f^{2}(\xi+S)\right\rangle-\langle f(\xi+S)\rangle^{2} \approx$ $\left\langle f^{2}(\xi)\right\rangle-\langle f(\xi)\rangle^{2}$. Note that this constraint also depends on the nonlinear function itself. In our experiment, the assumption is reasonably valid when the noise power is 1 order of magnitude larger than the input signal power. Equation (2) suggests that a linear function is appropriate for the Gaussian noise. A Gaussian noise gives the maximum entropy for a fixed noise variance. This means that there is no choice of transfer functions to reduce a Gaussian noise. Thus, the most appropriate function is one that transfers the signal without distortion: a linear function. On the other hand, if the noise is non-Gaussian, this shows that there is a suitable nonlinear function that provides an SNR that is better than that of the 
linear function. There exists an appropriate nonlinear function that maximizes the entropy of the noise.

The FFO approach can also be applied to optimizing a parameter in a given nonlinear function. In most cases, a nonlinear phenomenon is described by a specific equation. To control the behavior of the system, we often need to control a parameter in the given equation. As an example, consider a threshold function, $f_{\text {th }}$, such as a single step function or a sigmoid function: $f_{\text {th }}(x \geqslant \theta)=1$ and $f_{\text {th }}(x<\theta)=0$. The parameter that should be optimized is the threshold value $\theta$. The threshold function is known to cause SR, and the optimum threshold value has been discussed for specific noises in terms of this phenomenon [10,12]. Note that, in the case of Gaussian noise, the SR in the static threshold function cannot exceed the response in the linear function from Eq. (2). However, in the case of non-Gaussian noise, the threshold function has a positive possibility of exceeding the linear system. The FFO approach is applicable to a general noise for which the PDF has been identified. When $S$ is small enough, the output signal is expressed as $S\left\langle d f_{\text {th }}(\xi-\theta) / d \xi\right\rangle=S \rho(\theta)$. We let $P_{+}$denote the probability that the level of the output is high. Then the resulting SNR of the output is

$$
\mathrm{SNR}=\frac{S^{2} \rho^{2}(\theta)}{P_{+}\left(1-P_{+}\right)}, P_{+}=\int_{\theta}^{\infty} \rho(x) d x .
$$

We find the optimum threshold value when the SNR is maximized, that is, $\delta \mathrm{SNR} / \delta \theta=0$. Therefore,

$$
2 \frac{d \rho(\theta)}{d \theta}+\rho^{2}(\theta) \frac{1-2 P_{+}}{P_{+}\left(1-P_{+}\right)}=0 .
$$

The solution of this equation provides the optimum threshold value, which depends only on the PDF of the noise. When the dependence of $P_{+}$on $\theta$ is small, the SNR has a configuration similar to $\rho^{2}(\theta)$, and the maximum peak position of $\rho^{2}(\theta)$ provides the optimum $\theta$.

\section{EXPERIMENT}

We used an electronic system to examine the feasibility of the FFO-based design and optimization. For a small target signal, we used a sinusoidal wave with a frequency of $1 \mathrm{kHz}$ and an amplitude of $50 \mathrm{mV}$. For the non-Gaussian noise, we used a Gaussian mixture (as described above) generated by adding a PRBS and a Gaussian noise, both from conventional function generators. The bit rate of the PRBS was $1 \mathrm{Mbit} / \mathrm{s}$ and the amplitude of the bit was $1.27 \mathrm{~V}$. The bandwidth and the standard deviation of the Gaussian noise component were $1 \mathrm{MHz}$ and $0.32 \mathrm{~V}$, respectively. The noise was biased at $2.02 \mathrm{~V}$. Compared to the signal frequency, the noise can be considered white. Figure 1(a) shows the wave form of the measured noise. A histogram of the noise is shown in Fig. 1(b). The theoretical formula of the PDF, $\rho(x)=\left\{0.5 \exp \left[\left(x-\mu_{1}\right)^{2} / 2 V_{\mathrm{rms}}^{2}\right]+\right.$ $\left.0.5 \exp \left[\left(x-\mu_{2}\right)^{2} / 2 V_{\mathrm{rms}}^{2}\right]\right\} / \sqrt{2 \pi V_{\mathrm{rms}}^{2}}$, where $\mu_{1}$ and $\mu_{2}$ are the peak positions of the two Gaussian noise components and $V_{\mathrm{rms}}{ }^{2}$ is the variance of each component, was a good fit. The peaks of the PDF were observed when the noise voltages were 1.38 and $2.65 \mathrm{~V}$. The nonlinear functions were implemented using commercial electronic devices. The input and output wave forms were obtained by a sampling oscilloscope. The sampling rate was 10 times larger than either the bandwidth of the (a)

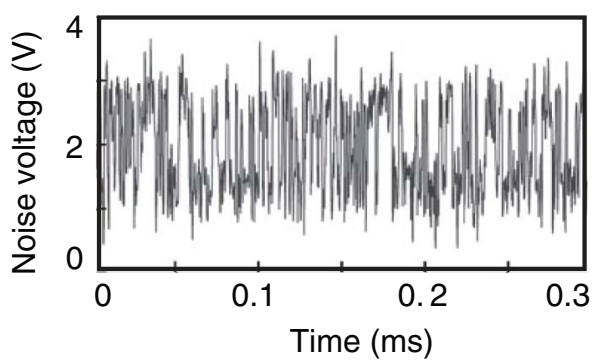

(b)

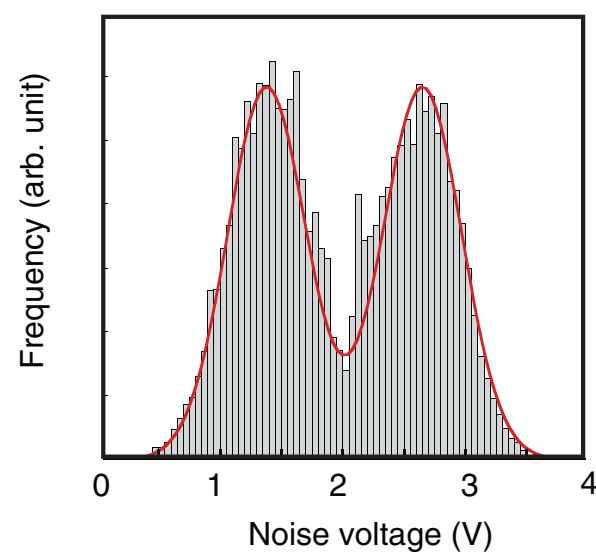

FIG. 1. (Color online) Generated mixed noise: (a) wave form and (b) histogram. Solid curve shows the theoretical probability density function.

Gaussian noise or the bit rate of the PRBS. The response was evaluated by determining the correlation coefficient between the target signal and the output wave forms. The total length of the time series was $20 \mathrm{~ms}$. Thus 20 periods of the input signal were used for characterization.

\section{RESULTS AND DISCUSSION}

\section{A. Optimum nonlinear function}

For the noise in Fig. 1, the optimum nonlinear function was designed using Eq. (2). The theoretical transfer curve obtained from the analytical PDF curve in Fig. 1(b) is shown as a broken line in Fig. 2. The curve has a negative slope near the input voltage $V_{\text {in }}$, which was $2 \mathrm{~V}$; in other regions, it is linear. This transfer characteristic was electronically implemented using a logic inverter and two resistors, as shown in the inset of Fig. 2. The measured curve from this device is shown as a solid line in Fig. 2. This device reasonably represented the designed characteristic, with only a small discrepancy around the region of the negative slope. The device was able to respond to input signals of over $1 \mathrm{MHz}$.

Examples of the measured input and output wave forms are shown in Fig. 3. The input for the nonlinear system was the sum of the target sinusoidal wave and the noise. We also considered this the output of the linear system. In the output of the nonlinear system shown in Fig. 3(a), the sinusoidal wave can be seen in the envelope and it was more obvious than it was in the linear system. To quantify this difference, we evaluated Pearson's correlation coefficient $C_{1}$ between the target signal and the output wave forms. For the nonlinear system, $C_{1}=0.16$, which was larger than that of 


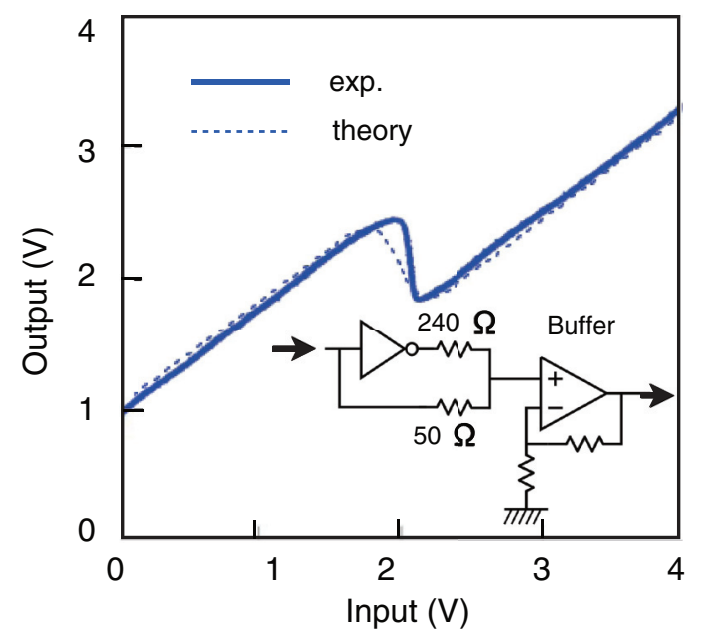

FIG. 2. (Color online) Theoretical and experimental transfer curves of the optimized nonlinear function for the mixed noise. Inset shows the electronic circuit for implementation of the function.

the linear system, 0.07. This difference is clearly seen in the filtered output wave forms, as shown in Fig. 3(b). The cutoff frequency of the filter $f_{C}$ was $10 \mathrm{kHz}$. Filtering was performed with a low-pass filter (LPF) by computing moving averages on the sampled data. The length of the moving average window was 1000 data points for $10-\mathrm{M}$ samplings/s data, which corresponds to time lengths from $100 \mu \mathrm{s}$. The nonlinear function suppresses the noise in the bandwidth of the target signal, but the noise outside the band remains. The LPF suppresses the noise outside the signal bandwidth and clarifies the effect of the nonlinear function. The nonlinear system with the LPF was able to recover the original input signal much better than did the linear system with the LPF. The input-output correlation coefficients after filtering were 0.92 and 0.64 for the nonlinear and linear systems, respectively. These results demonstrate the feasibility of the FFO-based design.

To clarify the effect of low-pass filtering on nonlinear output, we evaluated the correlation coefficient as a function of the LPF cutoff frequency $f_{\mathrm{C}}$. The results are shown in Fig. 4. The correlation coefficient greatly increased as the cutoff frequency decreased. The curve had a peak, since the (a)

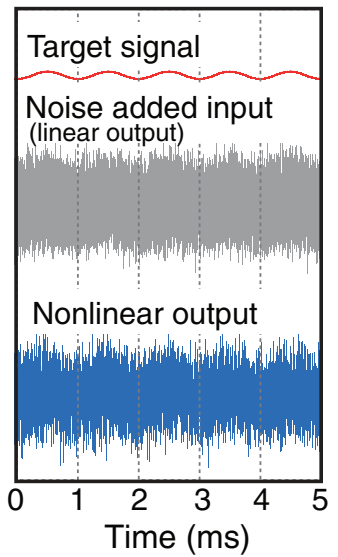

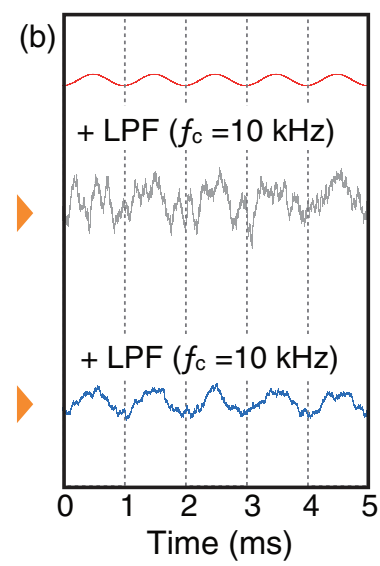

FIG. 3. (Color online) Examples of the measured input and output wave forms (a) before filtering and (b) after filtering.

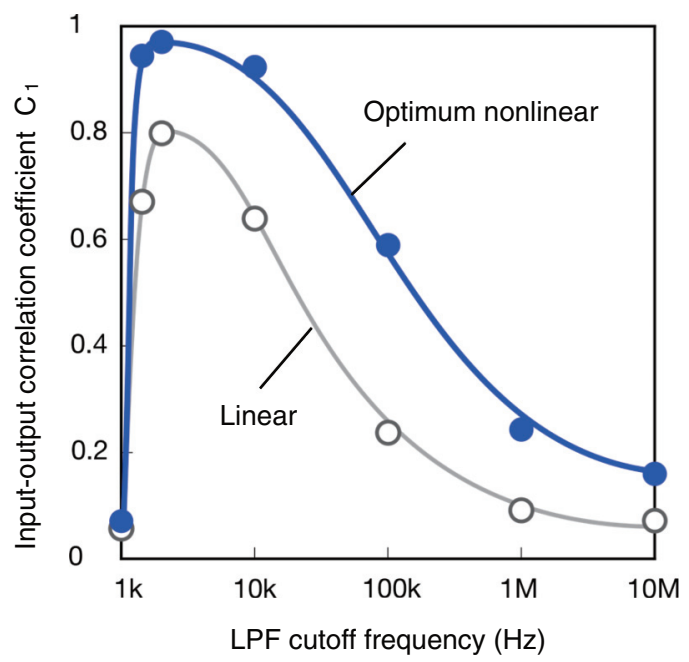

FIG. 4. (Color online) The input-output correlation coefficient as a function of LPF cutoff frequency for the optimum nonlinear and linear systems.

target signal itself was filtered when $f_{\mathrm{C}}$ was close to the frequency of the target signal. The filtered output of the linear system showed a similar curve; however, the correlation of the nonlinear system was always greater than that of the linear system. This result indicated that, for the present configuration of signal and noise, the performance of the optimum nonlinear function is superior. In terms of enhancing the response of a system, the benefit of the nonlinear function is acquired by the combination with an appropriate filtering. As shown in Fig. 4, we cannot achieve a peak value near unity with the correlation coefficient without the optimized nonlinear function.

The fact that the nonlinear system for the mixed noise performs better can be understood by considering the compression of the PRBS component through the Z-shaped negative transfer characteristic. This function can suppress the noise component existing within the bandwidth of the input signal. The nonlinear function can be approximated by two linear functions: $f=0.83 V_{\text {in }}+0.91\left(V_{\text {in }}<2 \mathrm{~V}\right)$ and $f=0.83 V_{\text {in }}$ $\left(V_{\text {in }}>2 \mathrm{~V}\right)$. When the input is less than $2 \mathrm{~V}$ and the PRBS is at a low level, the nonlinear function offsets the output by $+0.91 \mathrm{~V}$. This offset almost cancels the PRBS component with the amplitude of $1.27 \mathrm{~V}$. A margin between the offset and the PRBS amplitude arises from the $V_{\text {rms }}$ of the Gaussian noise component. The above process results in an output that consists of a target signal with a nearly Gaussian noise, and the system behaves like a linear system. In a conventional linear system, it is obvious that the SNR of the output with Gaussian noise is better than that with a mixture of the same Gaussian noise and a PRBS, since the power of the noise is increased by adding the PRBS. However, the SNR of the optimum nonlinear function with the mixed noise is somewhat smaller than that of the linear system with Gaussian noise. This is because the PDF of the Gaussian noise has long tails, which causes error in the switching of the two curves: for example, the high offset curve is selected even when the PRBS is at a high level. We should mention that the FFO-based design optimizes the function that includes this effect. In this study, the $V_{\text {rms }}(0.32 \mathrm{~V})$ of the Gaussian noise was sufficiently smaller than the offset $(0.91 \mathrm{~V})$, and thus this error was small. 
(a)

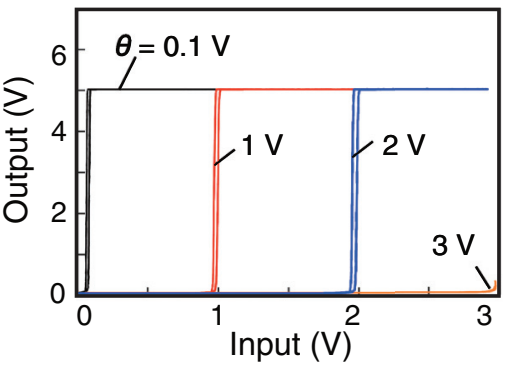

(b)

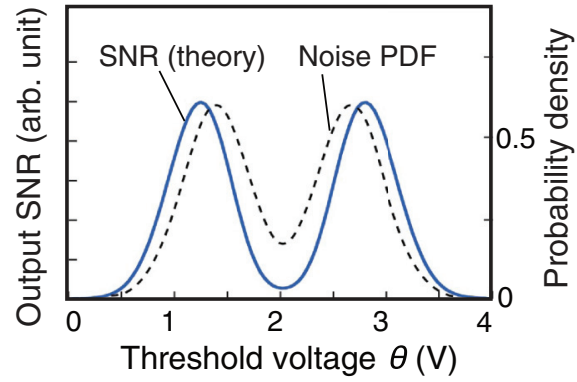

FIG. 5. (Color online) (a) Measured transfer characteristics of the threshold device in which the threshold value was systematically controlled. (b) Theoretical output SNR in the threshold function as a function of the threshold value. For comparison, the PDF of the mixed noise is also shown.

\section{B. Threshold function with optimized parameter}

Next we used FFO on the optimization of the threshold value of a threshold function. This parameter optimization should be useful because a practical system often needs to achieve a better response under a given transfer function, rather than completely designing a new function. We also considered the transmission of the weak signal with the mixed noise. A standard comparator was used as the threshold device. Figure 5(a) shows the measured transfer characteristics. The device exhibited a sharp threshold curve with a negligibly small hysteresis. The threshold value could be systematically controlled by the external voltage. Figure 5(b) shows the theoretical SNR for the mixed noise shown in Fig. 1, calculated from Eq. (3), as a function of the threshold value $\theta$. The curve of the estimated SNR of the output had a double peak, which is similar to the PDF of the mixed noise. We believe that this result is due to Eq. (3) being mainly dominated by $\rho^{2}(\theta)$ : the denominator on the right-hand side of the equation, corresponding to the standard deviation of the output noise, depends on the integral of $\rho$ and it changes slowly as a function of $\theta$. The positions of the peak provide the optimized threshold values that satisfy Eq. (4). We found two optimum threshold values, $\theta=1.24$ and $2.78 \mathrm{~V}$. The positions of the peak of the estimated SNR values shifted slightly away from the peaks in the noise PDF; this was due to $\theta$ dependence of the denominator on the right-hand side of Eq. (3).

Figure 6 shows examples of the measured input and output wave forms of the systems of the threshold function, with the threshold value of approximately the second peak $(\theta=3 \mathrm{~V})$ and the central valley of the estimated $\operatorname{SNR}(\theta=2 \mathrm{~V})$. The mixed noise shown in Fig. 1 was added to the signal. The output of the threshold system was composed to have an impulse train with the same amplitude. When $\theta=3 \mathrm{~V}$, the density of the

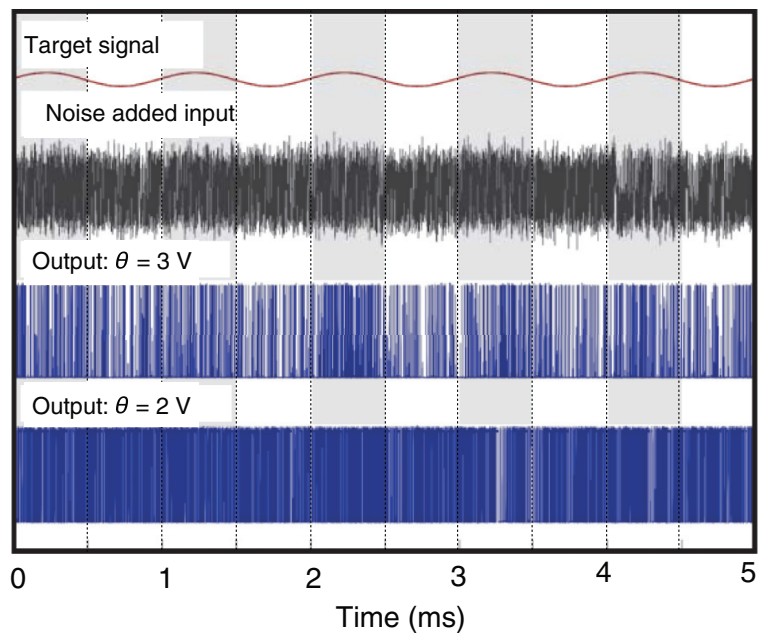

FIG. 6. (Color online) Measured input and output wave forms of the threshold device when the threshold was optimized $(\theta=3 \mathrm{~V})$ and not optimized $(\theta=2 \mathrm{~V})$. Gray hatched regions indicate the periods in which the target signal had a positive amplitude. When $\theta=3 \mathrm{~V}$, the pulse density was high in the gray region, indicating that the information in the target signal reflects the output.

impulses was modulated by the amplitude of the target signal. This behavior was similar to the pulse density modulation (PDM) or dither [3,19], where the amplitude of the target signal was converted to the density of the impulses. When $\theta=2 \mathrm{~V}$, a high-density impulse train was observed; however, the density seemed to be uniform. These results suggest that the efficiency of the information conversion depends on the threshold value.

We quantified the response of the threshold system by finding the correlation coefficient between the target signal and the output wave forms as a function of the threshold voltage. The result is shown in Fig. 7. To clarify the threshold dependence of the response, low-pass filtering with a cutoff frequency of $167 \mathrm{kHz}$ was performed on the output before calculating the correlation coefficient. This process corresponds to decoding the PDM signal, and thus the target signal is reproduced. The curve of the threshold system exhibited a double peak, as was expected from the theory. The theoretically predicted curve was calculated from the SNR in Fig. 5(b) using

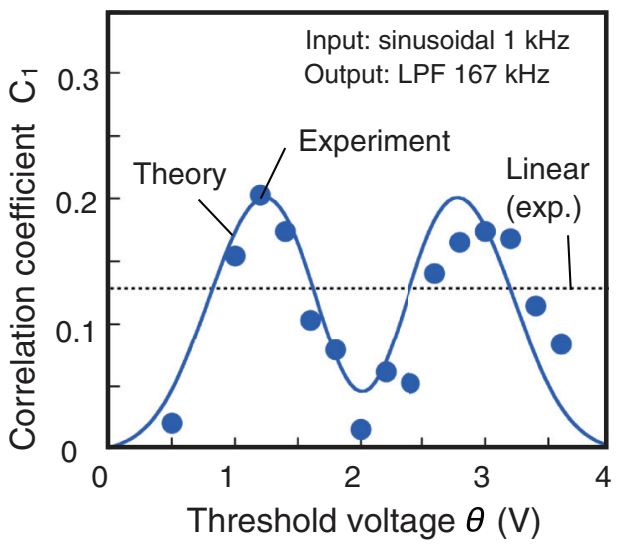

FIG. 7. (Color online) Input-output correlation coefficient for the threshold device as a function of the threshold value. 
an approximation formula, $C_{1} \sim(1+1 / \mathrm{SNR})^{-1 / 2}$; the plot is shown in Fig. 7. In this calculation, the amplitude of the target signal in Eq. (3) was adjusted to reflect the effect of the filtering; this barely changed the configuration of the curve. The results of the experiment were well explained by the theory and verified the validity of the FFO-based parameter optimization. The performance of the optimum nonlinear function is still better than that of the threshold function. The input-output correlation coefficients at a cutoff frequency of $167 \mathrm{kHz}$ for the optimum and threshold functions are 0.51 and 0.2 , respectively.

The correlation coefficient for the linear system is shown as a dashed line in Fig. 7. At the peaks, the correlation coefficient for the threshold system is larger than that for the linear system. The threshold system with the optimized $\theta$ better reproduced the target signal than did the linear system with noise. The superior performance of the simple threshold function for the mixed noise is thought to be due to the truncation of the PRBS component in the noise-added input, similar to that of the Z-shaped nonlinear function. In the noise used in this study, the power of the PRBS component $P_{\mathrm{PRBS}}$ was much larger than that of the Gaussian noise component $P_{\text {Gauss }}$, and the components were uncorrelated because they were generated independently. Thus the SNR in the linear system can be approximately evaluated by $P_{\mathrm{S}} /\left(P_{\mathrm{PRBS}}+P_{\mathrm{Gauss}}\right) \sim P_{\mathrm{S}} / P_{\mathrm{PRBS}}$ $\left(P_{\text {PRBS }} \gg P_{\text {Gauss }}\right)$. On the other hand, the threshold system omits the PRBS component when the threshold is adjusted to a high level of the PRBS components; this occurs at the second peak of the PDF of the noise. Thus the system effectively responds to the signal only with Gaussian noise. This effect can also be obtained when the threshold is adjusted to the first peak of the PDF of the noise. The SNR of the output [Eq. (3)] at the second peak of the PDF of the noise can be approximated by $(4 / 3 \pi) P_{\mathrm{S}} / P_{\mathrm{Gauss}}$. In the present model, the output noise is evaluated by the variance of the output of the nonlinear function. We assume the small input signal, and then the denominators of Eqs. (1) and (3) correspond to the power of the output fluctuation. We measured the correlation coefficient as a function of $P_{\text {Gauss }}$ at large $P_{\mathrm{PRBS}}$ and found that the correlation in the threshold system decreased as $P_{\text {Gauss }}$ increased, whereas that in the linear system was small and almost independent of $P_{\text {Gauss }}$. For $P_{\text {PRBS }} \gg P_{\text {Gauss }}$, the SNR in the threshold system was higher than that in the linear system.

The difference in the threshold point between the fully designed optimum function and the threshold step function arises from the difference in the details of the information representation. Using the step function, the signal information is represented with the density of the output impulse train. The information transfer efficiency is maximized when the threshold is adjusted to the point at which the change of the PDF of the noise by the input is the largest. This increases the output SNR in terms of increasing the numerator in Eq. (1). Considering Eqs. (1) and (2), the noise PDF with two similar peaks (as shown in Fig. 2) results in two optimal points. In the case of the optimum Z-shaped function, the impulses in the output are weighted through the finite slope in the outside of the negative slope region. This operation enhances the transmission of the input signal information owing to modulating not only the impulse density but also the amplitude. In addition, the negative slope around the threshold compresses the PRBS component of the noise, which also increases the output SNR by decreasing the denominator in Eq. (1). These two additional effects are boosted when the threshold point is at the center of the symmetric PDF of the noise: when the threshold is adjusted at one peak of the noise PDF, this function amplifies the noise in another peak, resulting in the decrease of the output SNR.

The present method can be applied to any kind of noise when the probability density function of the noise $\rho$ is known in advance and the noise intensity is larger than that of the input signal. The improvement factor of the response depends on the noise characteristics. From Eq. (1), in the case that the output noise power is constant-namely, the denominator of Eq. (1) is the same value-the output SNR increases as the derivative of the transfer function $f$ becomes large. The derivative of $f$ is approximately evaluated by $f^{\prime} \sim \rho^{-2}(\partial \rho / \partial x)^{2}-\rho^{-1}\left(\partial^{2} \rho / \partial x^{2}\right)$. This formula suggests that even with the same noise power, the noise having the large derivative at the inflection point of results in the large SNR in the present method. We should also mention that when the noise PDF has a complicated form, the implementation of the nonlinear function is difficult, which will be a constraint in the present method.

\section{CONCLUSION}

We designed a nonlinear function based on the functional Fisher optimization (FFO) approach to transfer a small signal buried in a non-Gaussian noise. We also used the FFO approach to optimize the threshold parameter in the threshold function for non-Gaussian noise. We electronically implemented the nonlinear functions and experimentally found that both nonlinear functions performed better than the linear system, demonstrating the feasibility of the FFO approach. The mechanism for the better response of the nonlinear functions is understood by the selective compression of the noise component that carries the smallest amount of information about the target signal.
[1] L. Gammaitoni, P. Hanggi, P. Jung, and F. Marchesoni, Rev. Mod. Phys. 70, 223 (1998).

[2] M. I. Dykman and P. V. E. McClintock, Nature (London) 391, 344 (1998).

[3] K. Wiesenfeld and F. Moss, Nature (London) 373, 33 (1995).

[4] N. G. Stocks, Phys. Rev. Lett. 84, 2310 (2000).
[5] S. Kai, T. Kai, M. Tanaka, and K. Hirakawa, J. Phys. Soc. Jpn. 47, 1379 (1979).

[6] X. Yu and E. R. Lewis, IEEE Trans. Biomed. Eng. 46, 36 (1989).

[7] R. N. Mantegna and B. Spagnolo, Phys. Rev. Lett. 76, 563 (1996).

[8] K. Murali, S. Sinha, W. L. Ditto, and A. R. Bulsara, Phys. Rev. Lett. 102, 104101 (2009). 
[9] B. Ando and S. Graziani, Stochastic Resonance: Theory and Applications (Kluwer Academic Publisher, Dordrecht, 2000).

[10] B. Kosko and S. Mitaim, Phys. Rev. E 70, 031911 (2004).

[11] D. Nozaki, D. J. Mar, P. Grigg, and J. J. Collins, Phys. Rev. Lett. 82, 2402 (1999).

[12] P. Jung, Phys. Lett. A 207, 93 (1995).

[13] A. Ichiki and Y. Tadokoro, Phys. Rev. E 87, 012124 (2013).

[14] R. J. Kozick and B. M. Sadler, IEEE Trans. Signal Processing 48, 3520 (2000).
[15] V. Bhatia and B. Mulgrew, Signal Processing 87, 2569 (2007).

[16] X. Wang and H. Vincent Poor, IEEE Trans. Signal Processing 47, 289 (1999).

[17] E. Abreu, M. Lightstone, S. K. Mitra, and K. Arakawa, IEEE Image Processing 5, 1012 (1996).

[18] R. A. Vazquez and H. Sossa, Neurocomputing 74, 2985 (2011).

[19] L. Gammaitoni, Phys. Rev. E 52, 4691 (1995). 